\title{
ESTUDO ANATÓMICO DA INERVAÇÃO DA PORÇÃO DISTAL DO MEMBRO TORÁCICO EM EQÜINO
}

\author{
ANATOMICAL STUDY OF THE INERVATION OF THE DISTAL PORTION \\ OF THE THORACIC LIMB IN HORSE
}

\section{Edson Moreira Borges ${ }^{1}$ Maria Verónica de Souza ${ }^{2}$ Tarcisio António Rego de Paula ${ }^{3}$}

RESUMO

Foram utilizados membros torácicos de 20 cavalos adultos de raça mestiça, provenientes do matadouro de equinos de Campo Belo - MG. Os nervos palmares medial e lateral, digitais palmares medial e lateral, e o ramo dorsal do nervo digital palmar (medial e lateral) foram dissecados para observação da freqüência de suas posições e ramificações acessórias. Os resultados demonstraram que o local de bifurcação do nervo palmar em ramo digital palmar e seu ramo dorsal, ocorreu com maior frequência na região proximal da articulação metacarpofalangeana tanto no membro torácico direito como no esquerdo e em ambas as faces medial e lateral. Foram constatados 5 tipos de padrões de divisão do nervo palmar, sendo o tipo 1 o predominante.

Palavras-chave: anestesia, bloqueio nervoso, inervação do membro torácico eqüino.

\section{SUMMARY}

In the se experiment were utilized 20 adult equine forelimbs, crossbred, collected in an equine abbatoir in Campo Belo, MG. Both lateral and medial palmar nerves, lateral and medial palmar digital nerves and lateral and medial dorsal branch of the palmar digital nerve were dissected to observe the frequency of their positions and accessory ramifications. The results showed that the sile of bifurcation of the palmar nerve into palmar digital branch and its dorsal branch, occurred most frequently in the proximal region of the metacarpofalangeal joint, both at the right and left forelimbs in their medial and lateral faces. Five types of standards of divisions of the palmar nerve into palmar digital branch and its dorsal branch with predominance of the type one were observed.

Key words: anaesthesia, nerve block, inervation of the equine forelimb.

\section{INTRODUÇÃO}

A utilização de bloqueios anestésicos como meio diagnóstico de claudicação em equinos é bastante aceita na prática veterinária, sendo benéfica nos casos em que, mesmo após uma anamnese eficiente e um acurado exame físico, a origem e a região específica desta claudicação ainda não foi identificada (DYSON, 1986; GIBSON e STASHAK, 1989; KEG et al., 1992).

Ocasionalmente, a claudicação no equino está relacionada com duas ou mais etiologias, e pode ser difícil decidir qual a lesão predominante com base apenas no exame físico (GIBSON \& STASHAK, 1989). Assim, com a utilização sistemática de bloqueios anestésicos pode-se encontrar a origem principal da maioria das claudicações.

Para que se obtenha melhores resultados, a anestesia local deverá ser realizada sistematicamente,

\footnotetext{
${ }^{1}$ Médico Veterinário, Aluno do Curso de Especialização em Medicina Veterinária, Universidade Federal de Viçosa (UFV).

${ }^{2}$ Médico Veterinário, Professora Assistente, Departamento de Veterinária, Área de Clínica de Grandes Animais, UFV, 36571-000, Viçosa - MG. Autor pra correspondência.

${ }^{3}$ Médico Veterinário, Professor Assistente, Departamento de Veterinária, Área de Anatomia dos Animais Domésticos, UFV.
} 
iniciando na porção mais distai do membro e progredindo proximalmente (GIBSON e STASHAK, 1989).

A expectativa após a realização desses bloqueios é que o animal deixará de claudicar apoiando temporariamente $\mathrm{o}$ membro afetadò quando a área que contém a lesão estiver dessensibilizada (DYSON, 1984). Entretanto, sabese porém que nem sempre melhoras ocorrem, pois existem inúmeros fatores que podem vir a interferir na eficiência desses bloqueios, tais como: localização inadequada do local de infiltração e principalmente variações anatómicas na distribuição nervosa. Na extremidade distai do membro torácico, essas variações consistem em geral, da existência de bifurcações e ramos acessórios dos nervos digitais palmares. Dessa forma poderá existir uma dessensibilização sem o desaparecimento da claudicação, apesar de se estar bloqueando o nervo no local recomendado (DYSON, 1984; 1986).

Os bloqueios mais utilizados ao nível da porção distai do membro torácico equino são: bloqueio nervoso digital palmar, bloqueio dos ramos dorsais dos nervos digitais palmares, bloqueio do nervo palmar ao nível dos ossos sesamóides proximais e bloqueio em quatro pontos (DYCE $\boldsymbol{e t}$ al., 1987; DYSON, 1991; DYSON \& KIDD, 1993; GIBSON \& STASHAK, 1989; STASHAK, 1994; VARNER, 1990).

Acreditando-se que as variações anatómicas na distribuição nervosa merecem maiores esclarecimentos decidiu-se realizar este trabalho que tem como objetivo, confirmar, observar o tipo e o local dessas variações ao nível do nervo palmar, nervo digital palmar e ramo dorsal do nervo digital palmar no membro torácico equino.

\section{MATERIAIS E MÉTODOS}

Foram utilizadas peças frescas (membros torácicos) de 20 cavalos mestiços, sendo 15 machos e 5 fêmeas, com idade entre 9 e 18 anos, com peso corporal variando entre 220 a $420 \mathrm{~kg}$, provenientes do matadouro de equinos em Campo Belo, MG. Os membros foram identificados e desarticulados na articulação rádio-uino-cárpica, logo após o abate eimediatamente após a colheita, o material foi congelado até a sua utilização. Após o descongelamento, toda a pele da peça foi retirada e os nervos palmares lateral e medial e digitais palmares lateral e medial com seus ramos dorsais foram dissecados para observação da constância de suas posições e ramificações acessórias. As peças dissecadas foram fotografadas e os trajetos dos nervos esquematizados para cada membro do animal, em ambas as faces, lateral e medial.
Os valores observados no experimento foram tabulados para variação percentual de posição e presença de ramificações nervosas acessórias.

\section{RESULTADOS E DISCUSSÃO}

Para descrever-se o local de bifurcação dos nervos palmares em nervos digitais palmares e seus ramos dorsais, tomou-se como referência, neste trabalho, a articulação metacarpofalangeana considerando-se: região proximal, região da articulação e região distai (Figura 1).

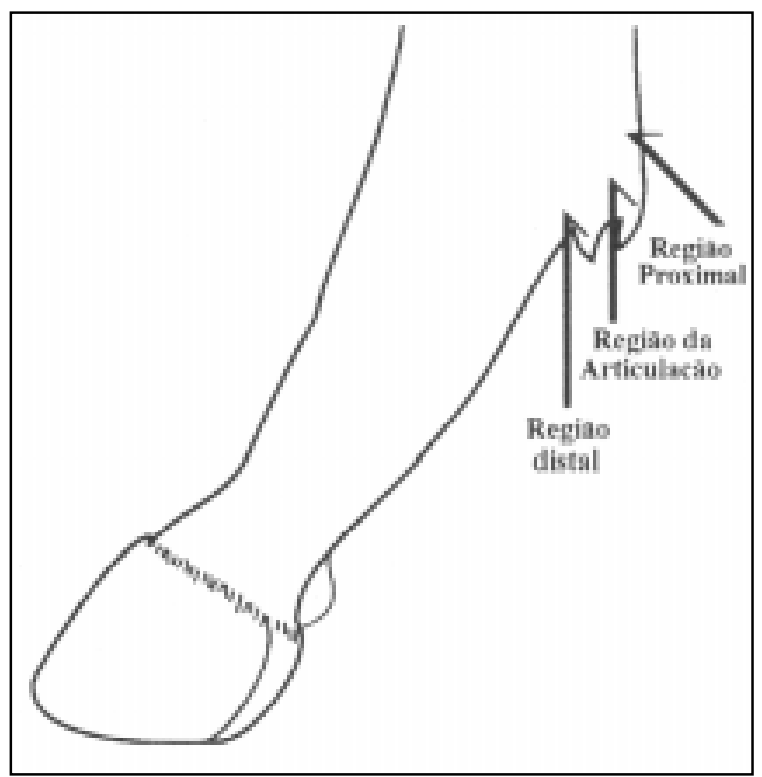

Figura 1 - Esquema da extremidade distal do membro torácico eqüino dividindo as regiões da articulações metacarpofalangeana em: região proximal, região articulação e região distal.

A bifurcação do nervo palmar em nervo digital palmar e ramo dorsal do nervo digital palmar ocorreu com maior frequência na região proximal da articulação metacarpofalangeana (Figura 2), tanto no membro torácico direito como no esquerdo, assim como nas suas faces medial e lateral (Tabela 1).

As Figuras 2, 3 e 4 mostram, respectivamente, a bifurcação do nervo palmar em nervo digital palmar e ramo dorsal do nervo digital palmar nas regiões proximal, da articulação e distai à articulação metacarpofalangeana.

Observou-se portanto, variações no local de bifurcação do nervo palmar em ramo digital palmar e seu ramo dorsal, porém na maioria dos casos (70 a 80\%) esta bifurcação ocorreu a nível da região proxi- 
mal da articulação metacarpofalangeana, o que demonstra uma certa constância, e o que nos leva a concordar com os achados de COLBERN (1984) e GETTY (1981), onde os autores afirmam que em geral esta bifurcação ocorre imediatamente proximal a articulação metacarpofalangeana.

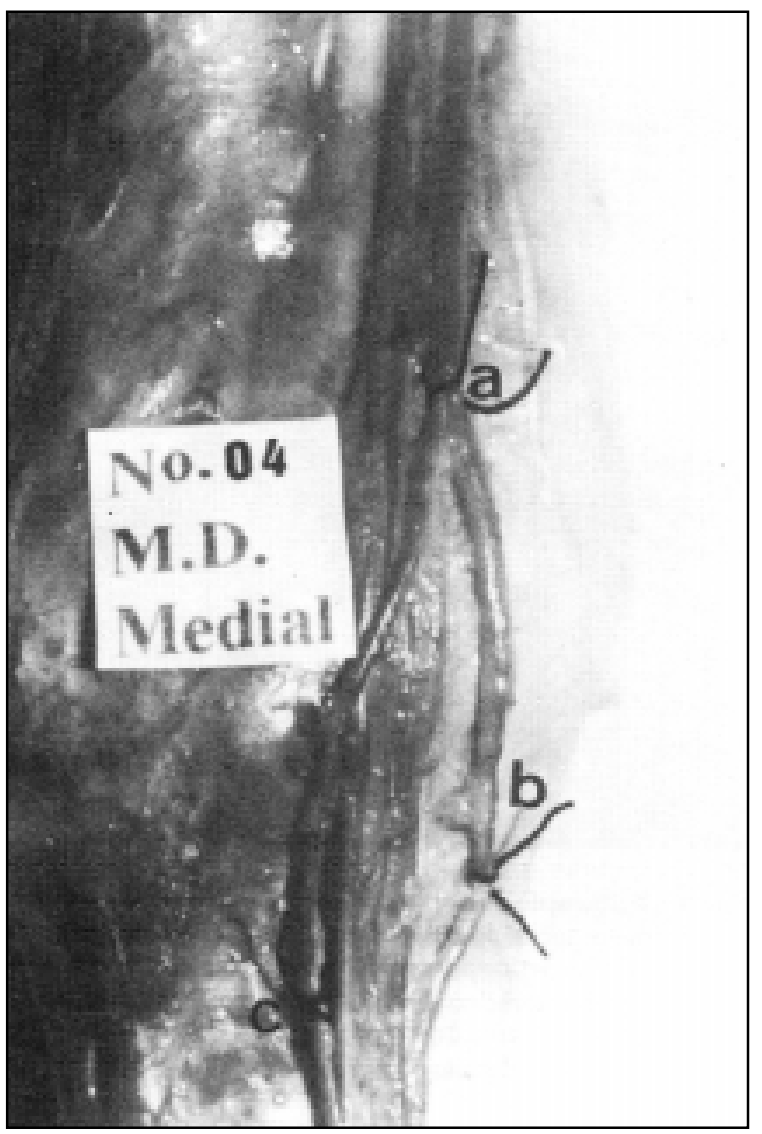

Figura 2 - Bifurcação do nervo palmar em nervo digital palmar e ramo dorsal do nervo digital palmar ao nível da região proximal da articulação metacarpofalangeana (cavalo $\mathrm{n}^{\circ} 4$ - membro torácico direito, face medial). a) nervo palmar medial; b) nervo digital palmar medial; c) ramo dorsal do nervo digital palmar medial.

Apesar de DYSON (1984, 1986) acreditarem que variações na distribuição nervosa são causas da não eficiência de um bloqueio nervoso, a falha num bloqueio em quatro pontos não pode ser devido as ramificações acessórias, uma vez que elas ocorrem apenas abaixo do ponto correio de aplicação anestésica, como indicado por STASHAK (1994). Por outro lado o mesmo não pode ser afirmado com relação ao bloqueio do nervo palmar ao nível dos ossos sesamóides proximais tendo em vista que o local para este bloqueio, segundo GIBSON \& STASHAK (1989) e VARNER (1990), é na superfície abaxial dos ossos sesamóides proximais. Durante a execução desta técnica pode-se não atingir o nervo palmar, mas os seus ramos: nervo digital palmar e/ou ramo dorsal do nervo digital palmar, que vão dessensibilizar apenas determinadas regiões anatómicas. Dessa forma este bloqueio deverá sempre ser realizado na região mais proximal possível dos ossos sesamóides proximais.

Tabela 1 - Local de bifurcação do nervo palmar em nervo digital palmar e ramo dorsal do nervo digital palmar no membro torácico direito e esquerdo de eqüino, nas faces lateral e medial.

\begin{tabular}{|c|c|c|c|c|}
\hline \multirow{2}{*}{$\begin{array}{l}\mathrm{N}^{\circ} \text { de } \\
\text { Animal }\end{array}$} & \multicolumn{2}{|c|}{ MTD } & \multicolumn{2}{|c|}{ MTE } \\
\hline & Medial & Lateral & Medial & Lateral \\
\hline 1 & $\mathrm{P}$ & $\mathrm{P}$ & $\mathrm{P}$ & $\mathrm{P}$ \\
\hline 2 & $\mathrm{P}$ & $\mathrm{P}$ & $\mathrm{P}$ & I \\
\hline 3 & $\mathrm{P}$ & $\mathrm{P}$ & $\mathrm{P}$ & $\mathrm{P}$ \\
\hline 4 & $\mathrm{P}$ & D & $\mathrm{P}$ & $\mathrm{D}$ \\
\hline 5 & $\mathrm{P}$ & $\mathrm{P}$ & $\mathrm{P}$ & $P$ \\
\hline 6 & $\mathrm{P}$ & $\mathrm{P}$ & $\mathrm{P}$ & $\mathrm{P}$ \\
\hline 7 & $\mathrm{P}$ & $\mathrm{P}$ & $\mathrm{P}$ & $\mathrm{P}$ \\
\hline 8 & $\mathrm{P}$ & $\mathrm{P}$ & $\mathrm{P}$ & $\mathrm{P}$ \\
\hline 9 & $\mathrm{P}$ & $P$ & $\mathrm{P}$ & $P$ \\
\hline 10 & I & D & $\mathrm{P}$ & $\mathrm{P}$ \\
\hline 11 & $\mathrm{P}$ & $\mathrm{P}$ & $\mathrm{P}$ & $\mathrm{P}$ \\
\hline 12 & I & D & $\mathrm{P}$ & D \\
\hline 13 & $\mathrm{P}$ & $\mathrm{P}$ & $\mathrm{P}$ & $\mathrm{P}$ \\
\hline 14 & $\mathrm{P}$ & $\mathrm{D}$ & $\mathrm{D}$ & $\mathrm{D}$ \\
\hline 15 & I & D & $\mathrm{P}$ & I \\
\hline 16 & $\mathrm{P}$ & $\mathrm{P}$ & $\mathrm{P}$ & $\mathrm{P}$ \\
\hline 17 & I & $\mathrm{P}$ & $\mathrm{D}$ & $\mathrm{P}$ \\
\hline 18 & $\mathrm{P}$ & $\mathrm{P}$ & $\mathrm{P}$ & $\mathrm{P}$ \\
\hline 19 & $\mathrm{D}$ & $\mathrm{P}$ & I & $\mathrm{P}$ \\
\hline 20 & I & $\mathrm{P}$ & D & $\mathrm{P}$ \\
\hline
\end{tabular}

Mtd - Membro Torácico Direito; MTE - Membro Torácico Esquerdo; P - Região Proximal da Articulação Metacarpofalangeana; I - Região da Articulação Metacarpofalangeana; D - Região Distal da Articulação Metacarpofalangeana.

Foram constatados 5 diferentes padrões de divisão do nervo palmar em nervo digital palmar e ramo dorsal do nervo digital palmar, cujos esquemas podem ser observados nas Figuras 5, 6, 7, 8 e 9. 


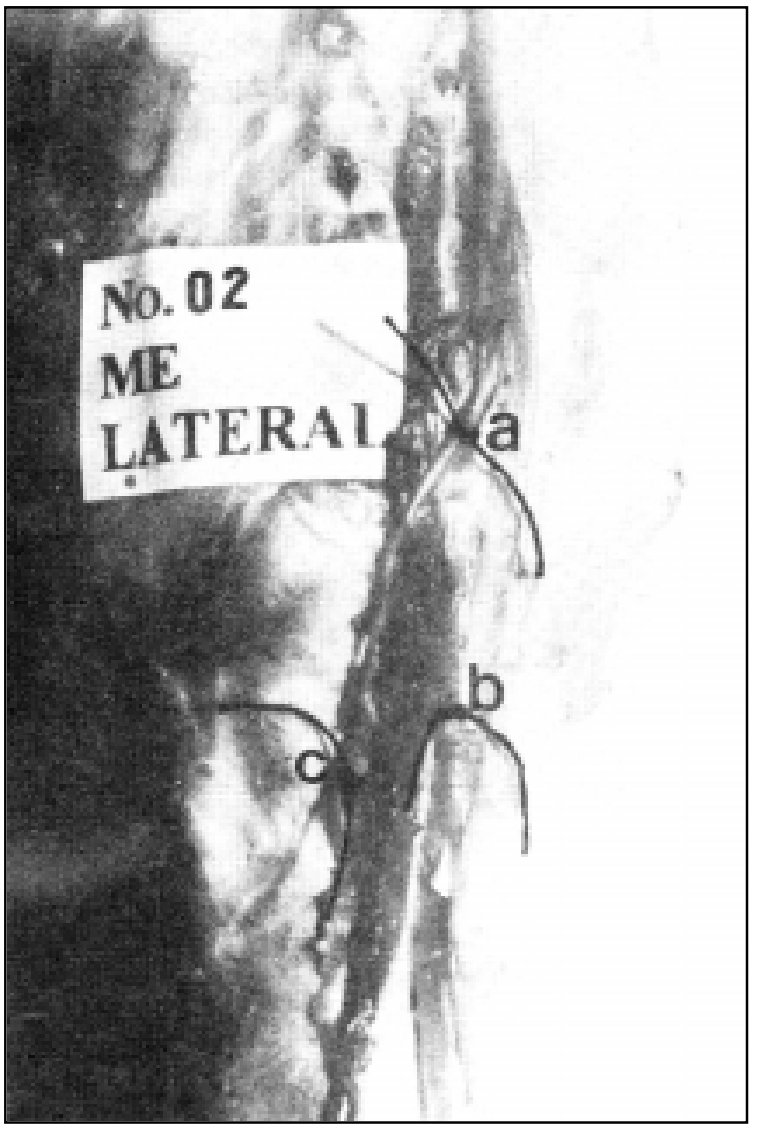

Figura 3 - Bifurcação do nervo palmar e ramo dorsal do nervo digital palmar ao nível da região da articulação metacarpofalangeana (cavalo $\mathrm{n}^{\circ} 2$ - membro torácico esquerdo, face lateral); a) nervo palmar lateral; b) nervo digital palmar lateral; c) ramo dorsal do nervo digital palmar lateral.

Na Tabela 2 pode-se constatar os tipos de variações anatómicas na distribuição nervosa no membro torácico equino direito e esquerdo, nas faces medial e lateral.

No membro torácico direito - face medial, $75 \%$ das amostras apresentaram padrão tipo $1 \mathrm{de}$ distribuição nervosa; $5 \%$ apresentaram padrão de divisão tipo 2 e $15 \%$ padrão de divisão tipo 3 . Ainda na face medial 5\% dos animais apresentaram o padrão 4 de divisão. No membro torácico direito, face lateral, $80 \%$ das amostras apresentaram padrão tipo 1 de distribuição nervosa; $5 \%$ padrão de divisão tipo 2; 5\% padrão de divisão tipo 4 e $5 \%$ padrão de divisão tipo 5.

No membro torácico esquerdo - face medial, $80 \%$ das amostras apresentaram padrão tipo 1 de distribuição nervosa; $5 \%$ padrão de divisão tipo $2 ; 15 \%$ padrão de divisão tipo 3 .

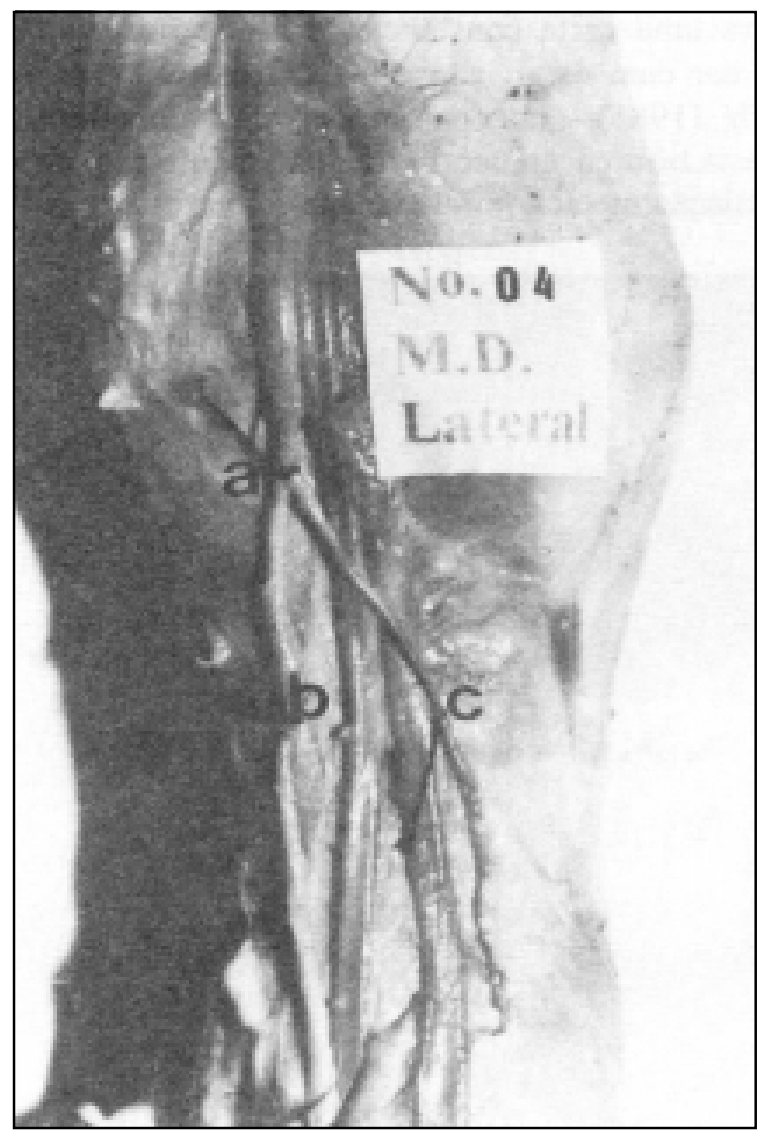

Figura 4 - Bifurcação do nervo palmar em nervo digital palmar e ramo dorsal do nervo digital palmar ao nível da região distal da articulação metacarpofalangeana (cavalo $n^{\circ} 4$ - membro torácico direito, face lateral); a) nervo palmar lateral; b) nervo digital palmar lateral; c) ramo dorsal do nervo digital palmar lateral.

No membro torácico esquerdo - face lateral, $85 \%$ das amostras apresentaram padrão tipo 1 de distribuição nervosa; $10 \%$ padrão de divisão tipo 3 e $5 \%$ padrão tipo 5 de divisão.

Apesar dos diferentes padrões de divisão encontrados em 75 a $85 \%$ dos casos, o padrão de divisão tipo 1 foi o predominante. Porém a ocorrência dos demais tipos de padrões de divisão pode vir a interferir na eficiência do bloqueio do nervo digital palmar e do ramo dorsal do nervo digital palmar, tendo em vista que nos padrões de divisão 3, 4 e 5 ocorre comunicação entre estes dois ramos. Portanto mesmo que o local de aplicação do anestésico, assim como a dose, estejam correios, o insucesso pode advir destas comunicações anatómicas, ainda pouco estudadas na literatura. $\mathrm{O}$ ramo dorsal do nervo digital palmar em geral lança vários ramos que podem não ser anestesiados durante a execução do bloqueio, o 


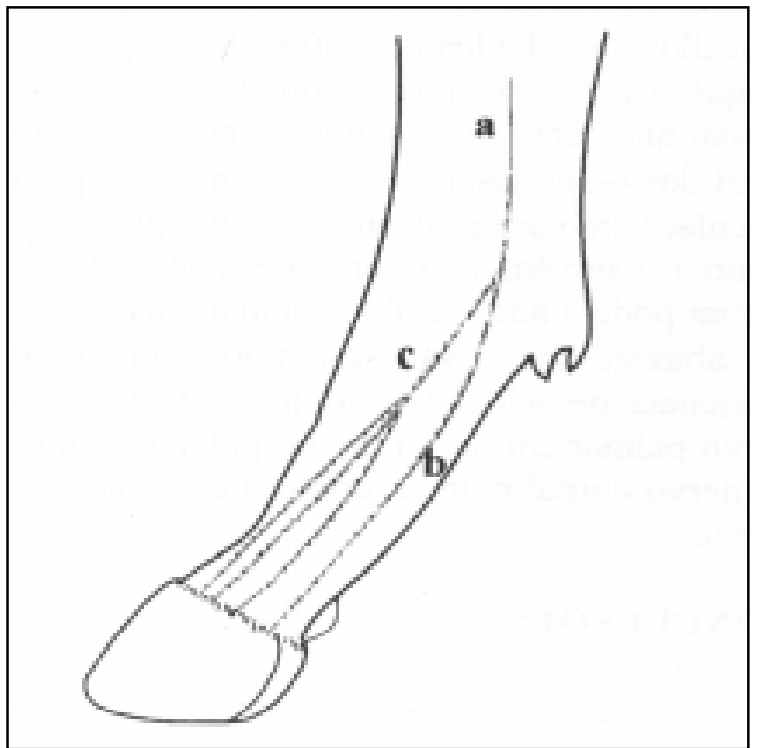

Figura 5 - Esquema do membro torácico esquerdo de eqüino, face lateral, demonstrando o padrão de divisão do nervo palmar em nervo digital palmar e ramo dorsal do nervo digital palmar. Tipo 1; a) nervo palmar lateral; b) nervo digital palmar lateral; c) ramo dorsal do nervo digital palmar lateral.

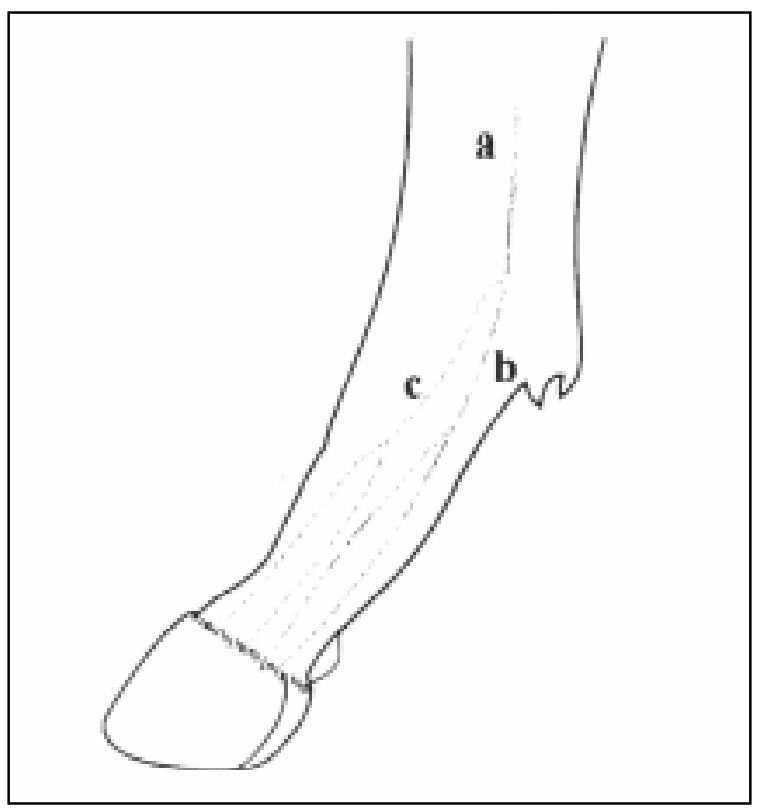

Figura 6 - Esquema do membro torácico direito de eqüino, face medial, demonstrando o padrão de divisão do nervo palmar em nervo digital palmar e ramo dorsal do nervo digital palmar. Tipo 2; a) nervo palmar medial;

b) nervo digital palmar medial; c) ramo dorsal do nervo digital palmar medial.

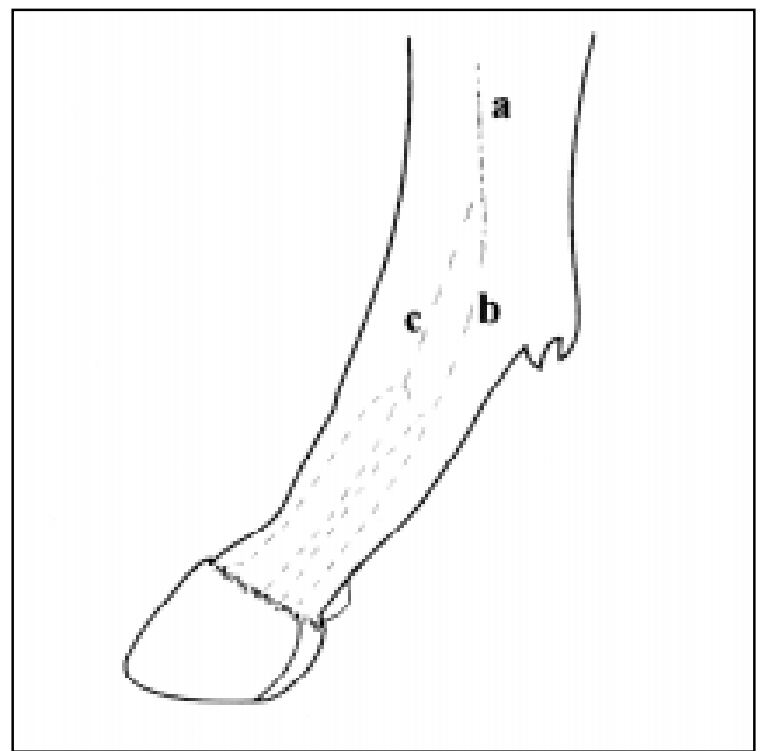

Figura 7 - Esquema do membro torácico direito de eqüino, face medial, demonstrando o padrão de divisão do nervo palmar em nervo digital palmar e ramo dorsal do nervo digital palmar. Tipo 3. a) nervo palmar medial; b) nervo digital medial; c) ramo dorsal do nervo digital palmar medial.

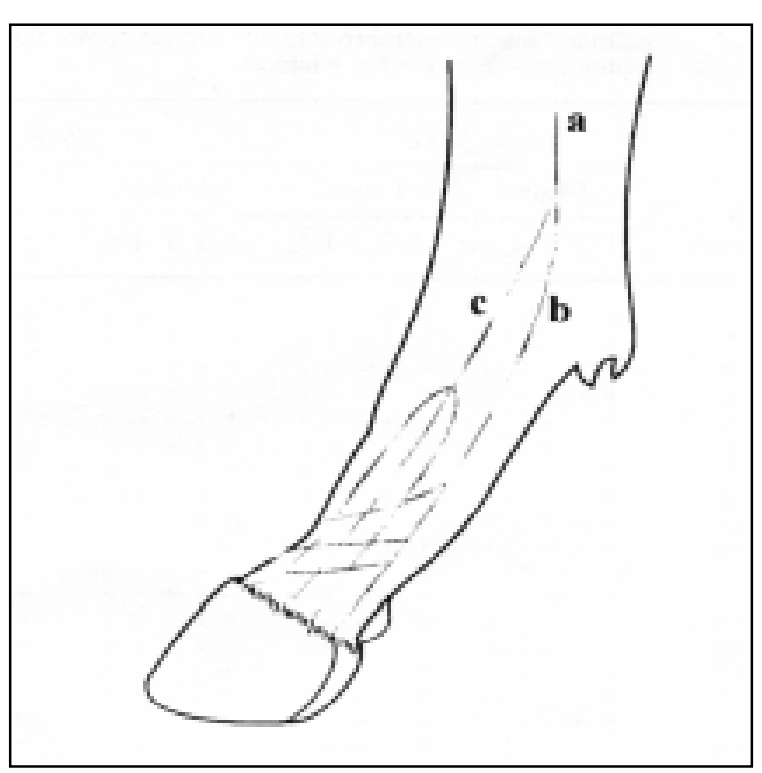

Figura 8 - Esquema do membro torácico direito de eqüino, face medial, demonstrando o padrão do nervo palmar em nervo digital palmar e ramo dorsal do nervo digital palmar. Tipo 4; a) nervo palmar medial; b) nervo digital palmar medial; c) ramo dorsal do nervo digital palmar medial. 


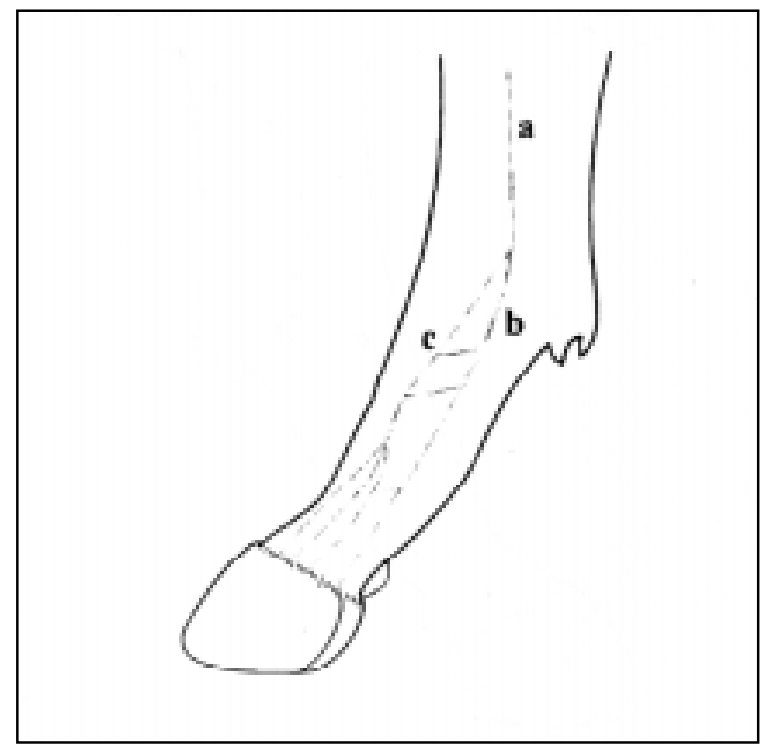

Figura 9 - Esquema do membro torácico esquerdo de eqüino, face lateral, demonstrando o padrão de divisão do nervo palmar em nervo digital palmar e ramo dorsal do nervo digital palmar. Tipo 5; a) nervo palmar lateral; b) nervo digital palmar lateral; c) ramo dorsal digital palmar lateral. mesmo acontecendo com o nervo digital palmar. Baseado nos achados deste trabalho sugere-se que o bloqueio nervoso mais adequado seria o do nervo palmar que como já mencionado pode ser realizado a nivel dos ossos sesamóides proximais ou proximal a articulação metacarpofalangeana (bloqueio em quatro pontos). Convém lembrar no entanto que, o nervo palmar poderá não ser bloqueado ao nível da superfície abaxial dos ossos sesamóides proximais pela existência de variações no local de bifurcação do nervo palmar em nervo digital palmar e ramo dorsal do nervo digital palmar, como mencionado anteriormente.

\section{CONCLUSÕES}

Os resultados encontrados nessa pesquisa demonstram que:

o local de bifurcação do nervo palmar em nervo digital palmar e ramo dorsal do nervo digital palmar ocorre com maior frequência na região proximal da articulação metacarpofalangeana;

existem pelo menos 5 tipos de padrões de divisão, com predominância do padrão de divisão tipo 1, tanto no membro torácico direito, como no esquerdo, em ambas as faces, lateral e medial.

\section{AGRADECIMENTOS} no membro torácico direito como no esquerdo de eqüino, nas faces medial e lateral.

\begin{tabular}{|c|c|c|c|c|}
\hline \multirow{3}{*}{$\begin{array}{l}\mathrm{N}^{\circ} \text { de } \\
\text { Animais }\end{array}$} & \multicolumn{2}{|c|}{ MTD } & \multicolumn{2}{|c|}{ MTE } \\
\hline & Medial & Lateral & Medial & Lateral \\
\hline & 12345 & 12345 & 12345 & 12345 \\
\hline 1 & $+\ldots$ & $+\ldots-$ & $+\ldots-$ & $+\ldots-$ \\
\hline 2 & $+\ldots-$ & $+\ldots-$ & $+\ldots-$ & $+\ldots-$ \\
\hline 3 & $+\ldots-$ & $+\ldots-$ & $--+\cdots$ & $+\ldots$ \\
\hline 4 & $+\ldots-$ & $+\ldots$ & $+\ldots-$ & $+\ldots$ \\
\hline 5 & $+\ldots-$ & --+ & $+\ldots-$ & $+\ldots-$ \\
\hline 6 & $+\ldots-$ & 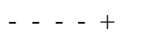 & $-+\cdots$ & $\ldots \ldots+$ \\
\hline 7 & $+\ldots-$ & $* \ldots$ & $+\ldots-$ & $+\ldots$ \\
\hline 8 & $+\ldots-$ & $+\ldots-$ & $+\ldots-$ & $+\ldots$ \\
\hline 9 & --+- & $+\ldots$ & $+\ldots-$ & $+\ldots$ \\
\hline 10 & --+- & $+\ldots$ & --+- & --+- \\
\hline 11 & $-\cdots+-$ & $+\ldots-$ & $+\ldots-$ & $+\ldots-$ \\
\hline 12 & $+\ldots-$ & $+\ldots$ & $-\cdots+-$ & $+\ldots$ \\
\hline 13 & --+- & $+\ldots$ & $+\ldots-$ & $+\ldots$ \\
\hline 14 & $+\ldots-$ & $+\ldots-$ & $+\ldots-$ & $+\ldots-$ \\
\hline 15 & $+\ldots-$ & $+\ldots-$ & $+\ldots-$ & --+-- \\
\hline 16 & $+\ldots-$ & $+\ldots-$ & $+\ldots-$ & $+\ldots-$ \\
\hline 17 & $+\ldots-$ & $+\ldots-$ & $+\ldots-$ & $+\ldots-$ \\
\hline 18 & $+\ldots-$ & $+\ldots$ & $+\ldots-$ & $+\ldots$ \\
\hline 19 & $+\ldots-$ & $+\ldots-$ & $+\ldots-$ & $+\ldots-$ \\
\hline 20 & -+-- & $+\ldots-$ & $+\ldots-$ & $+\ldots-$ \\
\hline
\end{tabular}

$\mathrm{N}^{\circ}$. Número do animal; 1,2,3,4 e 5: Tipo de padrão de divisão; +: Presença do padrão de divisão; -: Ausência do padrão de divisão; *: Padrão semelhante ao tipo 2 , porém sua bifurcação ocorre proximal a articulação metacarpofalangeana.
A CAPES e ao Hospital Veterinário Departamento de Veterinária da Universidade Federal Viçosa (DVT/UFV) pelo suporte financeiro .

Ao matadouro de equinos de Campo B MG pelo fornecimento dos animais.

\section{REFERÊNCIAS BIBLIOGRÁFICAS}

COLBERN, G.T. The use of diagnostic nerve block procedures on horses. Comp Contin Educ, v. 6, n. 1 O, p. 611-620, 1984.

DYCE, KM, SACK, W.O., WENSIMG, C.J.G. Tratado de anatomia veterinária. Rio de Janeiro: Guanabara Koogan, 1987. Cap. 23: o membro torácico do equino: p. 373-397.

DYSON, S. Nerve blocks and lameness diagnosis in the horse. In Pract, v. 6, n. 4, p. 102-107, 1984.

DYSON, S. Problems associated with the interpretation of the resulte of regional and intra-articular anaesthesia in the horse. Vet Rec, v. 118, p 419-422 1986.

DYSON, S. J. Lameness due to pain associated with the distai interphalangeal joint: 45 cases. Equine Vet J, v.23,n.2,p.128-135, 1991. 
DYSON, S.J., KIDD, L. A comparison of responses to analgesia of the navicular bursa and intra-articular analgesia of the distai interphalangeaijoint in 59 horses. Equine Vet J, v. 25, n. 2, p. 93-98, 1993.

GETTY, R. Anatomia dos animais domésticos. 5. ed. Rio de Janeiro: Interamericana, 1981. Cap. 24: sistema nervoso: p. 608-633.

GIBSON, K.T., STASHAK, T.S. Using perineural anesthesia to localize equine lameness. Vet Med, v. 84, n. 11, p. 1082, 1084-1086, 1989.

KEG, P.R., BELT, A.J.M. Van den., MERKENS, H.W. et al. The effeet of regional nerve blocks on the lameness caused by collagenase induced tendonitis in the midmetacarpal region of the horse: a study using gait analysis and ultrassonography to determine tendon healing. J Vet Med, v.39, n.5, p.349-363, 1992.

STASHAK, T. Claudicação em equinos segundo Adams. São Paulo: Roca, 1994. Cap. 3: diagnóstico de claudicação: p. 99159.

VARNER, D. D. Equine medicine and surgery. 4. ed. Califórnia: American Veterinary, 1990. Cap. 12: diseases of the musculoskeletal system: p. 1143-1538. 